\section{The Therapeutic Effect of Acupuncture Treatment on Chronic Inflammation of High Fat Diet Induced Rats}

\section{Abstract}

Objective: Obesity may be viewed as a chronic low-grade inflammatory disease, and there are increasing evidences proved that the acupuncture has the effect of anti-obesity; here we explored the therapeutic effect of acupuncture treatment on chronic Inflammation of high fat diet induced rats.

Methods: Use the high fat diet to induced obesity rats, and treat the rats with specific acupoints acupuncture and non-acupoints acupuncture. After the interference, all the rats were exposure to $\mathrm{CO}_{2}$ atmosphere and were killed by decapitation, the epididymis fat and the perirenal fat were isolated and weighted, and measured the serum level of FFA, TG, TC, IL- 6 and TNF $\alpha$. Results. The highfat-diet induced rats had heavier body weight, epididymal and perirenal fat pad weight, and higher level of FFA, TC, TG, IL- 6 and TNF $\alpha$ level in serum than the normal control group fed with standard chow. The specific acupoints and nonacupoints acupuncture treatment has the effect of significantly reducing body weight, epididymal and perirenal fat pad weight and serum FFA, TC, TG, IL-6 and TNF $\alpha$. Compare the specific acupoints and non-acupoints acupuncture treatment, the body weight, and epididymal and perirenal fat pad weight in specific acupoints treatment group lower than the non-acupoints treatment group, however there is no significant difference of serum FFA, TC, TG, IL-6 and TNF $\alpha$.

Conclusion: The specific acupoints (St 25, St 36, Sp6, P6 and St 40) acupuncture treatment have remarkable effect of anti-obesity and its mechanism may related to down-regulating the concentration of serum FFAs, IL-6 and TNF $\alpha$.

Keywords: Obesity; Acupuncture; Chronic inflammation

\author{
Meng Xia, Ling-Feng Jiang, \\ Dan-Dan Chen, \\ Zhao-Yang Zhang, \\ Hong-Teng Huang, \\ Mei Liang, Jia Liu and \\ Hong-Zhen Tang
}

Guangxi University of Chinese Medicine, Nanning 530001, China

Corresponding author: Hong-Zhen Tang

\section{6@qq.com}

Guangxi University of Chinese Medicine, Nanning 530001, China

Citation: Xia M, Jiang L, Chen D, et al. The Therapeutic Effect of Acupuncture Treatment on Chronic Inflammation of High Fat Diet Induced Rats. Herb Med. 2015, 2:1.

Received: November 07, 2015; Accepted: December 12, 2015; Published: December 22, 2015

\section{Background}

Obesity is a medical condition in which excess body fat has accumulated to the extent that it may have a negative effect on health [1-3], leading to reduced life expectancy and/or increased health problems, the major adverse effects include diabetes, heart disease, sleep apnea and other problems [4,5]. In fact, some researchers believe that obesity is second only to smoking as a preventable cause of death [6]. The use of western anti-obesity drugs has been limited by side effects including mood changes, suicidal thoughts, and gastrointestinal or cardiovascular complications [7-9]. The effectiveness and safety of acupuncture provide an alternative established therapy for this medical challenge. As early as in the Han dynasty (BC 202-
220), Chinese medicine practitioners have reported symptoms, mechanisms and risk factors of obesity and used acupuncture to control body weight. Acupuncture is the stimulation of specific acupuncture points along the skin of the body by thin needles, is one of the most popular types of traditional therapy. The multiple mechanisms of the efficacy of acupuncture have been attributed to the analgesic and anti-inflammatory effects $[10,11]$. Acupuncture is rapidly gaining recognition as an effective way to manage pain and discomfort $[12,13]$. There are more studies appearing that acupuncture is an effective way for treating obesity and this therapy is worldwide growing [14-16].

Base from the original theory of Chinese medicine, the key mechanism of obesity are "deficiency in origin and enrichment 
in symptoms" and/or "deficiency of spleen Qi and over-strong of stomach Qi". The patients with obesity gaining weight is because the deficiency of origin leads to the incompletely dispersing the nourishment and the un-thoroughly consuming of the energy which store in the adipocyte, the over much unburden fat brings about the enrichment complication including fatigue, hypertension, hyperglycemia, steatohepatitis. And what's more, in the traditional Chinese medicine theory, spleen and stomach are not what we usually named in western medicine. Spleen and stomach is the center of the whole body, spleen and stomach Qi play vital important roles in the movement of Qi. The orientation of the spleen qi movement is up and the orientation of stomach qi movement is down. The opposite direction of movement of spleen and stomach Qi balance the whole body' function. The spleen qi is to help disperse the nourishment; however the stomach $Q i$ is to help receiving food or digesting food. Thus, deficiency of spleen Qi and over-strong of stomach Qi leads to eat too much but cannot expend the energy properly.

Under the direction of this theory, we proposed that restoring the origin and strengthening spleen Qi and harmonizing the stomach Qi will rebalance the movement of $Q i$ and push the body back to health. In previous studies [17-20], we use acupoints zusanli, sanyinjiao, taishu, fenglong and zhongwan. To balance spleen Qi and stomach Qi, which showed a significant reduced body index for rats of obesity or over weight. And considering the obesity is a kind of status of chronic inflammation $[3,7,21]$ we speculate on the role of cytokines in our understanding of the links between anti-inflammatory activity and the effectiveness of acupuncture for obesity treatment and may uncover the acupuncture mechanism for the prevention and treatment of obesity.

\section{Materials and Methods}

\section{Ethics Statement}

All protocols were approved by the institutional animal care and use committee of Guangxi University of Chinese Medicine.

\section{Animals and diets}

50 Adult male (50-60 days) Sprague-Dawley rats (160-200 g) were purchased from Hunan Slack Scene of Laboratory Animal Co. Ltd. (Hunan, China). All animals were housed 5 animals per plastic cages with soft bedding on a $12 \mathrm{~h} \sim 12 \mathrm{~h}$ light-dark cycle (light cycle: 08:00 20:00) and at a constant temperature (22 \pm $\left.2^{\circ} \mathrm{C}\right)$ and humidity $(50 \pm 10 \%)$. The high fat diet were fed to 40 rats based on literature [22] containing 7\% (wt:wt) fat using Corn Oil (COO), Rapeseed oil (RSO), or lard (Ssniff). Rats received high fat diets and water ad libitum. 10 control rats received only the regular diet containing COO. Body weights were recorded once a week. After 8 weeks, determined the successfully obesity rats as their body weight excessed the average weight of the rats fed with regular diet. And then, select 30 successful obesity rats from the 40 rats fed with high fat diets. And then, all the 30 obesity rats and 10 control rats were separately administrated with different intervention. After 5 weeks of intervention, all the rats were feed-deprived overnight, sedated by exposure to $\mathrm{CO}_{2}$ atmosphere, and killed by decapitation. Blood was collected and processed for serum and the pididymisadipose and perirenal fats were collected.

\section{Experimental groups and the administration}

The experiment was designed for four groups: a) control with regular diet and situation (CTL), totally 10 rats. b) Nutritional obesity only (Nu.), totally 10 rats. c) Nutritional obesity following manual acupuncture on the specific acupoints (Nu.A.), totally 10 rats. d) Nutritional obesity following manual acupuncture on non-acupoints (NA-For), totally 10 rats. In the control group, rats were bonded and tied with the same duration as the other groups under the same conditions.

\section{Acupuncture needle and manual acupuncture}

All experimental needles were provided by Ruikang Hospital affiliated to Guangxi University of Chinese Medicine (Nanning, China). We used stainless steel needles $(0.3 \mathrm{~mm}$ in diameter and $30 \mathrm{~mm}$ in length, Huatuo Sterile Needles, Suzhou, China). The acupoints of zusanli (ST36), Tianshu (St-25), Fenglong (S40), Zhongwan (CV12), Yinlinquan (SP9), and Guanyuan (CV4) were selected to use in the experiments. The Zusanli (ST36) acupoint is located between the tibia and fibula at approximately $5 \mathrm{~mm}$ lateral and $5 \mathrm{~mm}$ lower to the anterior tubercle of the tibia [23]. Tianshu (St-25), the only bilateral point of this series, is located on a horizontal line 5 Tsun above the symphysis pubis and 2 Tsun lateral to the midline (right and left) [24]. All the acupoints location and the needles applying to these acupoints were shown in Figure 1. A lipid tissue found along the border of the body's trunk at the thigh or at the abdomen on the ipsilateral side was selected as our non-acupoints. A stainless-steel needle was inserted into the appropriate acupoint by twirling two times clockwise and counter-clockwise at each second. Such twirling continued for $1 \mathrm{~min}$ and paused for $5 \mathrm{~min}$ for 5 times which took a total of $30 \mathrm{~min}$. St-25, CV12, CV4 acupoints and their related non-acupoint insertion was at a depth of $4 \sim 5 \mathrm{~mm}$, while the ST36, S40, SP9 acupoints and the related non-acupoint depth insertion was $2 \sim 3 \mathrm{~mm}$ (Figure 1B). Rats received acupuncture every other day and continuous for constant 35 days, whereas the control rats received only presses without a needle. All the interference begins at 7 weeks' diet.

\section{Measurements of serum triglyceride, total cholesterol, IL-6 and TNF $\alpha$ levels}

Serum samples from the rats were maintained at $-80^{\circ} \mathrm{C}$ until analysis. Levels of triglyceride, total cholesterol, IL- 6 and TNF $\alpha$ were measured using an enzyme-linked immunosorbent assay kit according to the manufacturer's method (Boster, Wuhan, China).

\section{Statistical analysis}

The results presented reflect means \pm SD per group. A one way analysis of variance (ANOVA) was used to compare the body weight, $\mathrm{BMI}$, the serum triglyceride, total cholesterol after acupuncture intervene, serum IL-6 and TNF $\alpha$ levels. Less than 0.05 was considered statistically significant (SPSS 14.0, SPSS Inc., Chicago, Illinois, USA). 

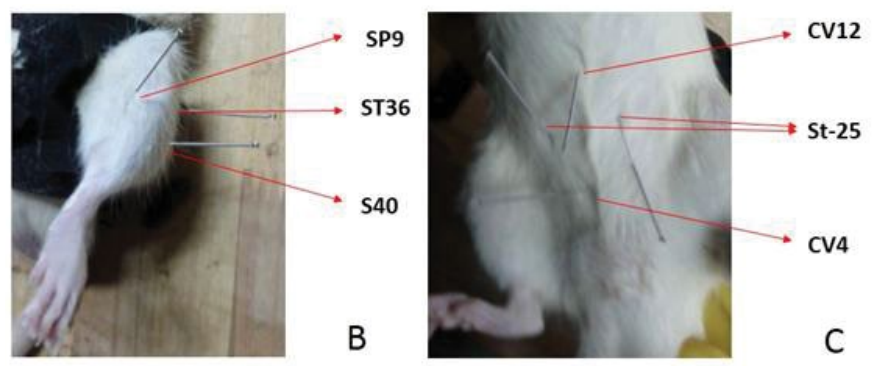

Figure 1

Acupuncture needle and manual acupuncture. All rats received high fat diets and water ad libitum expect controls rats; However, control rats received. The regular diet for continuous 7 weeks. From the $8^{\text {th }}$ week rat were separately treated with specific acupoints. Acupuncture, nonacupunture and only pressed.

\section{Results}

\section{Body weight were measured every week throughout the experiments}

Body weights were measured every week throughout the experiment. At week 6 , the beginning of body weight of group $\mathrm{Nu}$, Nu.A, NA-FOR weighted significantly more $(n=8, p<0.01$ one way ANOVA) than control rats. At week 7, the differences were further increase and it showed the MAX difference. And during the first 7 weeks, body weight increased steadily in all the four groups (CTL, Nu, Nu.A, NA-FOR). At week 8 , rats of group Nu.A and NAFOR received acupuncture every other day and continuous for constant 35 days, whereas the control rats received only presses without a needle. The body weight of Nu still steadily increased weekly but not as fast as the first 7 weeks (the duration when the rats were feed with diet). The body weight of group Nu.A, NA-FOR begun to decrease and the rats of group Nu.A decrease faster than them in NA-FOR group. And at week 12, the body weight of group Nu.A significantly lower than Nu, NA-FOR and control group (Figure 2).

After the interference, all the rats were exposure to $\mathrm{CO}_{2}$ atmosphere and were killed by decapitation, the epididymis fat and the perirenal fat were isolated and weighted. The pididymisadipose mass and the perirenal fat of Nu group significantly higher than control group $(P<0.05)$. The treatment of acupuncture decreased the pididymisadipose mass and the perirenal fat, and the pididymisadipose mass and the perirenal fat of Nu.A group were statically significantly lower than them in Nu group $(P<0.05)$. Even the NA-For group, the pididymisadipose mass and the perirenal fat also significantly lower than them in Nu group $(P<0.05)$ but significantly higher than control group $(P<0.05)$. There were no significantly difference of pididymisadipose mass and the perirenal fat between Nu.A group and NA-For group $(P>0.05)$ (Figure 3).

Blood was collected by cardiac puncture after the rats were killed under $\mathrm{CO}_{2}$ anesthesia at the end of the experiments. Serum was obtained by blood centrifugation at $4000 \mathrm{rpm}$ for 10 $\min$ at $4^{\circ} \mathrm{Cand}$ immediately stored at $-80^{\circ} \mathrm{C}$ for further analyses. Serum free fatty acids (FFA), triglyceride (TG), total cholesterol
(TC) were measured by enzymatic colorimetric methods using commercial kits from NanJing JianCheng bioengineering institute (Nanjing, China). Interleukin-6 (IL-6) and TNF $\alpha$ concentration were estimated by ELISA with a commercially available kit from Boster (Wuhan, China). The effect of acupuncture are shown in Figures $\mathbf{4}$ and $\mathbf{5}$. The Nutritional obesity group had significantly $(p<0.05)$ high level of FFA, TC and TG relative to the normal control rats. The serum FFA, TC and TG level were affected by the 35 days manual acupuncture on specific acupoints, while the nutritional obesity rats were followed with manual acupuncture on the specific acupoints more significantly reduced serum FFA, TC and TG level compared with nutritional obesity rats followed with manual acupuncture on the non-acupoints.

The serum IL-6 and TNF $\alpha$ showed the same tendency with the changes of FFA, TC and TG. The serum IL- 6 and TNF $\alpha$ level of nutritional obesity group had a significant difference compare with normal control group. And the acupuncture on the specific acupoints significantly reduced them. And there is statistical significantly difference between Nu.A group and NA-For group (Figure 5).

\section{Discussion}

In the present study, we observed that the high-fat-diet induced rats had heavier body weight, epididymal and perirenal fat pad weight, and higher level of FFA, TC, TG, IL- 6 and TNF $\alpha$ level in serum than the normal control group fed with standard chow. The administration of acupuncture on specific acupoints significantly reduced body weight, epididymal and perirenal fat pad, and serum FFA, TC, TG, IL-6 and TNF $\alpha$ level compared with the Nutritional obesity group fed with high fat diet. The results implicated that acupuncture on specific acupoints could
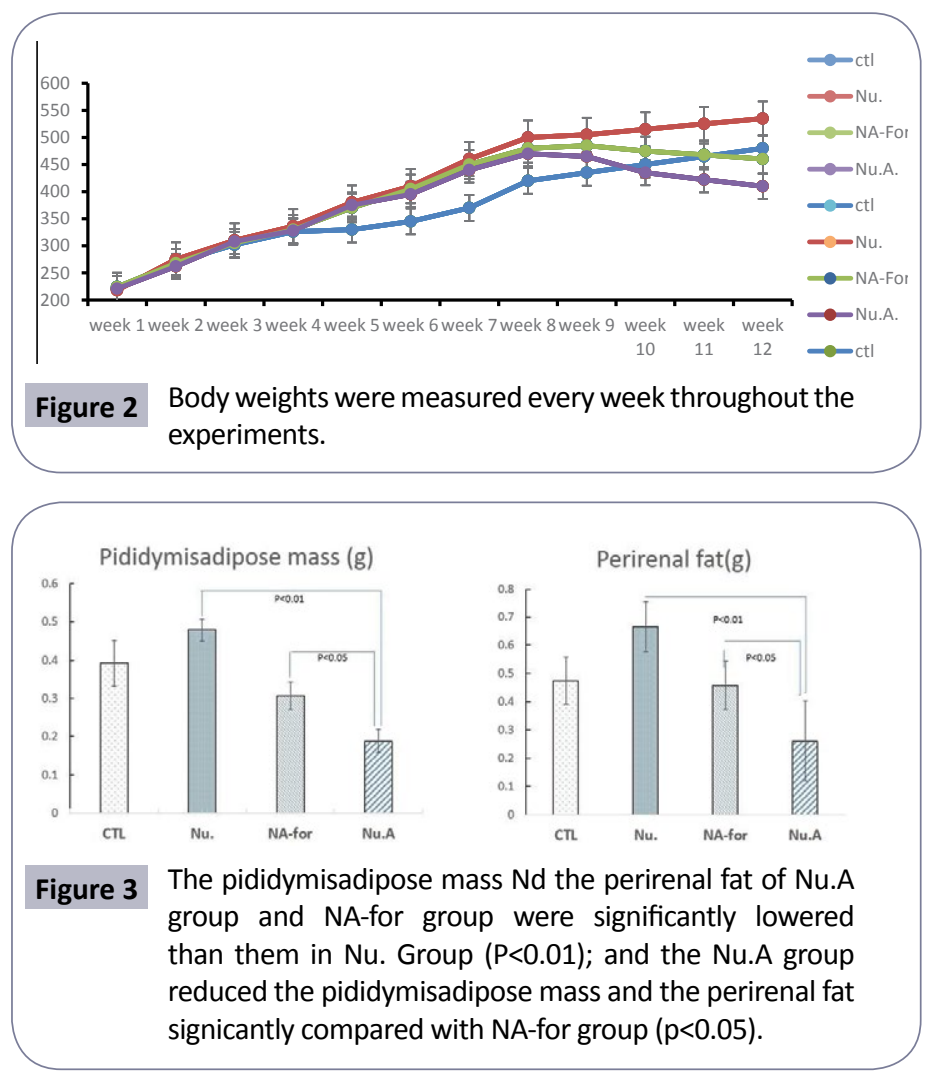


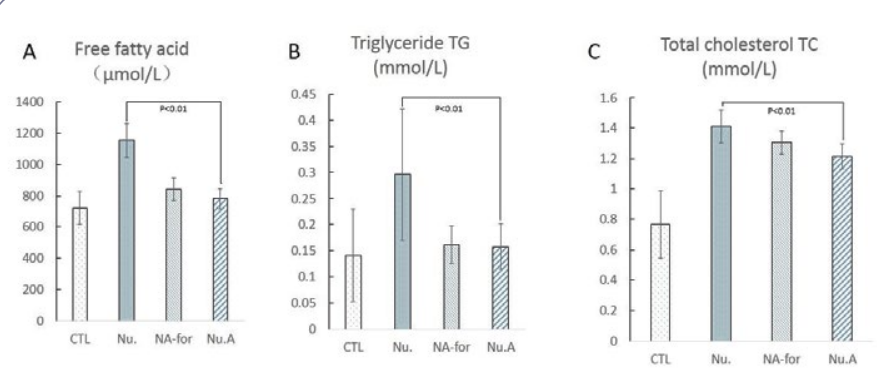

Figure 4 The FFa, TG, TC of Nu.A group were significantly lower than them in Nu. Group $(P<0.05)$ there were no significantly difference between Nu.A group and NA-for group $(p>0.05)$.

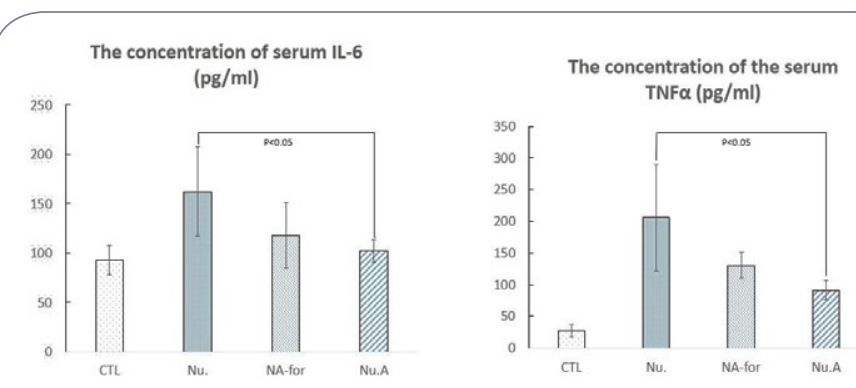

Figure 5 The level of serum IL-6 and TNF $\alpha$ were statically significantly lower than them in Nu. Group $(P<0.05)$ there were no significantly difference between Nu.A group and Na-for group ( $p>0.05$ ).

ameliorate the Inflammatory state of rats with high fat diet induced obesity.

There are increasing evidences proved that the acupuncture treatment is effective for body weight reduction in rats and humans [14-16]. Rong-Tsung Lin [25] reported that the plasma levels of FFA increased markedly in steroid-background rats, the stimulation of ST36 acupoint significantly decrease them. Cabioglu [26] investigated the effect of acupuncture therapy (Hegu (LI 4), Quchi (LI 11), Tianshu (St 25)) on body weight and found that the lipolytic effect of acupuncture may reduce the morbidity of obesity by mobilizing the energy stores that result in weight reduction. Neyhan [27] investigated the role of changes in Leptin and beta endorphin (BE) levels in weight loss following acupuncture application (St 25, St 36, Sp6, P6 and St 40) in obesity treatment and found that acupuncture application with diet restriction in obesity treatment is more effective than the diet restriction. We used the comprehensive acupoints including ST36, St-25, S40, CV12, SP9, and CV4, all the acupoint were used to treat obesity human or animals in literature. Previously, we observed that the acupuncture treatment on specific acupoints with ST36, St-25, S40, CV12, SP9, and CV4 significantly reduce the body weight, lee's index of high fat diet induced rats compared with non- treatment high fat diet induced rats, and these specific acupoints acupuncture treatment also have the effect of upregulating Hypothalamus ob-R mRNA expression [17-20]. In this project, we found that the specific acupoints acupuncture significantly reduced body weight, epididymal and perirenal fat pad weight of obesity rats and the results were consistent with previous results.

One of the greatest factors contributing to the obesity is high fat diet. Fat consumption has been found to be a key player in the obesity epidemic [28-30]. Long-term consumption of the high fat food can lead to obesity and consequently damaging effects on general health. Adipose tissue is an active endocrine organ; adipocytes control whole body energy homeostasis through the storage of triglycerides and release of fatty acids. Long term high fat food consumption increase the size of the adipocytes and the number of the adipocytes, and then high level of triglyceride (TG) and total cholesterol (TC) will be found in the serum. In the previous experiments [17-20] we examined the serum TG, TC, high density lipoprotein (HDL) and low density lipoprotein (LDL), and found that the high fat diet significantly increase serum TG, TC, LDL and decrease the serum HDL, however the specific acupoints acupuncture treatment reversed the effect and it significantly decrease the serum level of TG, TC, LDL and increased the serum level of HDL. The present results similar with these results. It implicated that the high fat diet successfully induce obesity fat rat model and the specific acupoints acupuncture have the effect of anti-obesity.

In obesity high levels of free fatty acids (FFA) contribute to inflammation. Numerous studies have reported that elevated adiposity is associated with increased plasma pro-inflammatory cytokines [31-33]. The excess storage of TAG from dietary intake results in an excessive influx of free fatty acids into blood circulation. And then, the free fatty acids leads to lowgrade inflammation characterized by over production of proinflammatory adipocytokines [34,35]. Adipocytes mediate inflammatory response by regulating the release of free fatty acids and adipocytokines, in particular tumor necrosis factor- $\alpha$ (TNF $\alpha$ ), interleukin-6 (IL-6). A recent study reported that the consumption of high fat mixed meal increased plasma IL- 6 concentration compared with control rats [36]. Previous works conducted a 6-week crossover dietary intervention with reduced energy diets in 15 overweight men and found that Plasma TNF $\alpha$, IL-6 concentrations did not differ between diets but appreciably reduced compared with baseline. In this study, the similar results were found: the concentration of FFAs, IL- 6 and TNF $\alpha$ significantly were up-regulated by high fat diet induced, and the specific acupuncture treatments have the effect of down-regulating the serum FFAs, IL-6 and TNF $\alpha$ concentration.

In the present project, we also compared the effect of specific acupoints and non-acupoint acupuncture treatment. The results showed that the non-acupoints acupuncture treat have significantly effect on reducing body weight, epididymal and perirenal fat pad weight, serum TC, TG, IL- 6 and TNF $\alpha$ level compared with Nutritional obesity rats. But compared with non-acupoints treatment, the specific acupoints base on the traditional Chinese medicine has a better effect on reducing body weight, epididymal and perirenal fat pad weight. However, the two different acupoints acupuncture treatment have a similar effect on reducing obesity's rats' serum FFA, TG, TC, IL-6 and TNF $\alpha$. It looks like that the specific acupoints and non-specific acupoints acupuncture have different mechanism on treatment 
and maybe that's why the specific acupoints acupuncture have a better effect on treating obesity. It still needs more evidences to proof that.

\section{Conclusion}

High fat diet provoke inflammatory response in obesity rats however the effect of types of dietary fats remained uncertain. The specific acupoints (St 25, St 36, Sp6, P6 and St 40) acupuncture treatment have remarkable effect of anti-obesity and its mechanism may related to down-regulating the concentration of serum FFAs, IL- 6 and TNF $\alpha$. The specific acupoints and nonacupoints acupuncture which base on the theory of Chinese medicine may have different mechanism on treating obesity. A complete understanding on the role of acupuncture treatment on low grade inflammation merits further investigation. Information is needed based on rigorously well designed clinical studies. 


\section{References}

1 Gremese E, Tolusso B, Gigante MR, Ferraccioli G (2014) Obesity as a Risk and Severity Factor in Rheumatic Diseases. Autoimmune Chronic Inflammatory Diseases. p: 576.

2 Murphy CC, Martin CF, Sandler RS (2014) Racial Differences in Obesity Measures and Risk of Colorectal Adenomas in a Large Screening Population. Nutrition and Cancer- An International Journal. pp: 1-7.

3 Gase LN, DeFosset AR, Smith LV, Kuo T (2014) The Association between Self-Reported Grocery Store Access, Fruit and Vegetable Intake, Sugar-Sweetened Beverage Consumption, and Obesity in a Racially Diverse, Low-Income Population. Front Public Healt 2: 229.

4 Swithers SE, Sample CH, Davidson TL (2013) Adverse effects of highintensity sweeteners on energy intake and weight control in male and obesity-prone female rats. Behavioral Neuroscience 127: 262-274.

5 Cooper-DeHoff RM, WenS, Beitelshees AL (2010) Impact of abdominal obesity on incidence of adverse metabolic effects associated with antihypertensive medications. Hypertension 55: 61-68.

6 Espey DK, Jim MA, Cobb N (2014) Leading causes of death and allcause mortality in American Indians and Alaska Natives. American Journal of Public Health 10: S303-311.

7 Habtemariam S (2012) The anti-obesity potential of sigmoidin A. Pharmaceutical Biology 50: 1519-1522.

8 Elkalmi R, Hassali MA, Al-Lela OQ, Jawad AA, Al-Shami AK, et al. (2013) Adverse drug reactions reporting: Knowledge and opinion of general public in Penang, Malaysia. J Pharm Bioallied Sci 5: 224-228.

9 Sui Y, Zhao HL, Wong VC (2012) A systematic review on use of Chinese medicine and acupuncture for treatment of obesity. Obes Rev 13: 409-430.

10 Yuan H, Chen R, Huang DP, Wang Y, Wang WY (2011) Analgesic and anti-inflammatory effects of balance acupuncture on experimental scapulohumeral periarthritis in rabbits. Zhongguo Zhen Jiu 31: 1106-1110.

11 Da SM, Bobinski F, Sato KL, Kolker SJ, Sluka KA, et al. (2014) IL-10 Cytokine Released from M2 Macrophages is Crucial for Analgesic and Anti-inflammatory Effects of Acupuncture in a Model of Inflammatory Muscle Pain. Molecular Neurobiology.

12 Li W, Cheng YH, Yu XG (2012) Observation on therapeutic effect of acupuncture combined with medicine on mild cognition disorders in patients with post-stroke. Zhongguo Zhen Jiu 32: 3-7.

13 Jiang YP, Liu H, Xu P, Wang Y, Lu GH, et al. (2011) Effect of electroacupuncture intervention on cognition attention bias in heroin addiction abstinence-a dot-probe-based event-related potential study. Chin J Integr Med 17: 267-271.

14 Ren BB, Liu ZC, Xu B (2012) Observation on the efficacy of female obesity complicated with climacteric syndrome treated by acupuncture and moxibustion. Zhongguo Zhen Jiu 32: 871-876.

$15 \mathrm{Wu}$ J, Li Q, Chen L, Tian D (2014) Clinical research on using acupuncture to treat female adult abdominal obesity with spleen deficiency and exuberant dampness. J Tradit Chin Med 34: 274-278.

16 Set T, Cayir Y, Pirim AB (2014) Effects of ear acupuncture therapy for obesity on the depression of obese women. Acupunct Med 32: 427-429.

17 Hongyang Li, Hong-Zhen Tang, Yong Tang (2012) The Effect of Specific Acupoints Acupuncture treatment on Obesity. Lishizhen Medicine And Materia Medica Research 06: 1466-1467.

18 Hong-Zhen T, Lan-Ying Z, Shi-Jun Y (2013) Influence of Specific Acupoint Acupuncture Therapy to Serum Angiogenesis Factor of
Obese Mouse Models. World Science and Technology/Modernization of Traditional Chinese Medicine and Materia Medica 06: 1370-1374.

19 Hong-yang Li, Hong-Zhen Tang (2011) Effect of Acupuncture on the Expression of Hypothalamus ob mRNA in a Rat Model of Obesity. China's basic medical journal of traditional Chinese medicine 04: 423-424.

20 Hong-Zhen T, Hong-Yang L, Yong P, Li-Fan L (2011) Effect of Acupuncture On The Serum Leptin/Insulin in a Rat Model of Obesity. Journal of Chengdu Medical College 03: 235-237.

21 Ramos N (2013) The role of chronic inflammation in obesityassociated cancers. ISRN Oncol: 697521.

22 Graf D, Barth SW, Bub A (2014) Dietary fat quality in regular fat diets has minor effects on biomarkers of inflammation in obese Zucker rats. European Journal of Nutrition 53: 211-219.

23 Wen YR, Yeh GC, Shyu BC (2007) A minimal stress model for the assessment of electroacupuncture analgesia in rats under halothane. European Journal of Pain 11: 733-742.

24 Sugai GC, Freire AO, Tabosa A, Yamamura Y, Tufik S, et al. (2004) Serotonin involvement in the electroacupuncture- and moxibustioninduced gastric emptying in rats. Physiology \& Behavior 82: 855-861.

25 Lin RT, Tzeng CY, Lee YC (2009) Acute effect of electroacupuncture at the Zusanli acupoints on decreasing insulin resistance as shown by lowering plasma free fatty acid levels in steroid-background male rats. BMC Complement Altern Med 9: 26.

26 Cabioglu MT, Ergene N (2005) Electroacupuncture therapy for weight loss reduces serum total cholesterol, triglycerides, and LDL cholesterol levels in obese women. Am J Chin Med 33: 525-533.

27 Cabioglu MT, Ergene N (2006) Changes in serum leptin and beta endorphin levels with weight loss by electroacupuncture and diet restriction in obesity treatment. Am J Chin Med 34: 1-11.

28 Fung TT, Rimm EB, Spiegelman D (2001) Association between dietary patterns and plasma biomarkers of obesity and cardiovascular disease risk. American Journal of Clinical Nutrition 73: 61-67.

29 Cordain L, Eaton SB, Sebastian A (2005) Origins and evolution of the Western diet: health implications for the 21st century. American Journal of Clinical Nutrition 81: 341-354.

30 Demigne C, Bloch-Faure M, Picard NM (2006) Chronically fed a westernized experimental diet as a model of obesity, metabolic syndrome and osteoporosis. European Journal of Nutrition 45: 298-306.

31 Olson NC, Callas PW, Hanley AJ (2012) Circulating levels of TNF-alpha are associated with impaired glucose tolerance, increased insulin resistance, and ethnicity: the Insulin Resistance Atherosclerosis Study. J Clin Endocrinol Metab 97: 1032-1040.

32 Bose KS, Gupta SK, Vyas P (2012) Adipocytokine levels in genetically high risk for type 2 diabetes in the Indian population: a crosssectional study. Exp Diabetes Res 2012: 386524.

33 Gelaye B, Revilla L, Lopez T (2010) Association between insulin resistance and C-reactive protein among Peruvian adults. Diabetol Metab Syndr 2: 30

34 Soto-Vaca A, Losso JN, McDonough K, Finley JW (2013) Differential effect of 14 free fatty acids in the expression of inflammation markers on human arterial coronary cells. J Agric Food Chem: 6110074-6110079.

35 Capurso C, Capurso A (2012) From excess adiposity to insulin resistance: the role of free fatty acids. Vascul Pharmacol 57: 91-97.

36 Phillips LK, Peake JM, Zhang X (2013) Postprandial total and HMW adiponectin following a high-fat meal in lean, obese and diabetic men. European Journal of Clinical Nutrition 67: 377-384. 